\title{
Ephrin Receptor A4 Expression Enhances Migration, Invasion and Neurotropism in Pancreatic Ductal Adenocarcinoma Cells
}

\author{
SATORU FURUHASHI ${ }^{1,2}$, YOSHIFUMI MORITA ${ }^{1}$, SHINYA IDA $^{1}$, RYUTA MURAKI $^{1}$, \\ RYO KITAJIMA ${ }^{1}$, MAKOTO TAKEDA ${ }^{1}$, HIROTOSHI KIKUCHI ${ }^{1}$, \\ YOSHIHIRO HIRAMATSU ${ }^{1,3}$, MITSUTOSHI SETOU ${ }^{2,4}$ and HIROYA TAKEUCHI ${ }^{1}$ \\ ${ }^{1}$ Department of Surgery, Hamamatsu University School of Medicine, Shizuoka, Japan; \\ ${ }^{2}$ International Mass Imaging Center and Department of Cellular and Molecular Anatomy, \\ Hamamatsu University School of Medicine, Shizuoka, Japan; \\ ${ }^{3}$ Department of Perioperative Functioning Care and Support, \\ Hamamatsu University School of Medicine, Shizuoka, Japan; \\ ${ }^{4}$ Preeminent Medical Photonics Education \& Research Center, \\ Hamamatsu University School of Medicine, Shizuoka, Japan
}

\begin{abstract}
Background/Aim: We sought to identify the mechanisms of perineural invasion in pancreatic ductal adenocarcinoma (PDAC). Materials and Methods: We utilized in vitro cancer cell-nerve co-culture models comprising human PDAC cell lines (MIA Paca2 and PANC1) and a dorsal root ganglion (DRG) isolated from neonatal mice. We compared gene expression profiles between cell lines with/without DRG conditioned medium (DRG-CM) using RNA-sequencing (RNA-seq). Results: Migration, invasion, and neurotropism were significantly enhanced in MIA Paca2 but not in PANC-1 cells co-cultured with DRGs. Among 285 genes which showed significant differences in expression levels between cell lines in RNA-seq, we focused on Ephrin receptor A4 (EPHA4), which was upregulated in MIA Paca2 cells treated with DRG-CM. The abilities of migration, invasion, and neurotropism enhanced by DRG coculture were abolished when EPHA4 was knocked down by siRNA in MIA Paca2 cells. Conclusion: EPHA4 can be a potential target gene to regulate perineural invasion in PDAC cells.
\end{abstract}

This article is freely accessible online.

Correspondence to: Yoshifumi Morita, MD, Ph.D., Department of Surgery, Hamamatsu University School of Medicine, 1-20-1 Handayama, Higashi-ku, Hamamatsu, Shizuoka, 431-3192, Japan. Tel: +81534352279/Fax: +81534352273, e-mail: yoshi-mo@hamamed.ac.jp

Key Words: Perineural invasion, pancreatic ductal adenocarcinoma, EPHA4, prognosis.
Pancreatic ductal adenocarcinoma (PDAC) is a devastating malignancy with dismal prognosis and ranks as the fourth highest cause of cancer-related death in the United States (1). Most patients with PDAC present with locoregional spread or metastatic disease at the time of diagnosis (2). Most cases recur even after local disease control has been achieved by surgical intervention and are strongly refractory to systemic chemotherapy (3). Thus, improved recognition of the biological behavior of PDAC, particularly the aggressive nature of its invasion, is urgently needed.

Perineural invasion (PNI) is a common pathological feature of PDAC, as evidenced by its high incidence observed in surgical specimens of $\operatorname{PDAC}(4,5)$. Previous reports have shown that PNI contributes to locally advanced and/or metastatic disease progression in patients with PDAC $(6,7)$. In the past, the anatomical proximity between the pancreas and the periarterial plexus has been implicated in the development of PNI in PDAC (8). More recently, it has been suggested on the basis of neurotropic theory, that the nerves and invading tumor cells interact with each other using neurotrophins to establish PNI $(9,10)$. However, the detailed molecular mechanisms of PNI in PDAC remain to be fully elucidated.

There are few ideal in vitro experimental models to study PNI in malignant disease. Dai et al. have established an in vitro co-culture model using a human PDAC cell line and mouse dorsal root ganglion (DRG), and demonstrated neurite outgrowth from the DRG and enhanced colony formation by PDAC cells, suggesting a mutual growth support (11). Our previous study has utilized this in vitro cancer cells-nerve cells co-culture model with modifications and investigated the role of Tenascin $\mathrm{C}$, an extracellular matrix protein, by evaluating the interactions between PDAC and nerve cells (12). 
In this study, we sought to identify the molecules in PDAC cells that are responsible for PNI. First, we evaluated the differences in the migration, invasion, and neurotropic abilities of PDAC cells using an in vitro cancer cells-nerve cells co-culture model. Second, using RNA sequencing (RNA-seq), we compared the gene expression profiles of two PDAC cell lines (MIA Paca2 and PANC-1) treated with or without DRG-conditioned medium (DRG-CM). Of these genes, Ephrin receptor A4 (EPHA4) was suggested to be a potential target gene that regulates PNI in PDAC cells.

\section{Materials and Methods}

Cell culture. The human pancreatic cancer cell lines, MIA Paca2 and PANC-1, were purchased from RIKEN Bioresources Cell Bank (BRC Cell Bank, Ibaraki, Japan) and provided by the Cell Resource Center for Biomedical Research, Tohoku University (Sendai, Japan). MIA Paca2 was routinely grown in complete Dulbecco's modified Eagle's medium (DMEM; Invitrogen, Carlsbad, CA, USA) supplemented with $10 \%$ Fetal Bovine Serum (FBS), at $37^{\circ} \mathrm{C}$ in a humidified atmosphere saturated with $5 \% \mathrm{CO}_{2}$. PANC-1 was grown in RPMI1640 (Wako, Osaka, Japan) with $10 \% \mathrm{FBS}$ at $37^{\circ} \mathrm{C}$ in a humidified atmosphere saturated with $5 \% \mathrm{CO}_{2}$.

Isolation of DRG from neonatal mice. The following animal procedures were performed according to the guidelines of the Committee on Experimental Animals of Hamamatsu University School of Medicine (17-323). The procedures of mouse DRGs' isolation were described by Ayala et al. (13). Briefly, neonatal (1day-old) ICR mice (Japan SLC, Shizuoka, Japan) were anesthetized by isoflurane and euthanized by cervical dislocation. Each DRG was isolated by performing an anterior laminectomy and microscopic dissection from the lumbar spinal region.

Migration and invasion assays using cancer cell-neuron vertical coculture model. In vitro cell migration and invasion were evaluated using a cell culture insert and a Matrigel invasion chamber (Becton Dickinson, Bedford, MA, USA) separated by an $8 \mu \mathrm{m}$-pore filter membrane in 24 well-plates. For the cancer cell-nerve vertical coculture model, four DRGs collected from neonatal mice were seeded in $20 \mu \mathrm{l}$ of Matrigel (\#356231, Matrigel ${ }^{\circledR}$ Growth Factor Reduced Basement Membrane Matrix, Corning, NY, USA) in the lower chamber, and incubated at $37^{\circ} \mathrm{C}$ saturated with $5 \% \mathrm{CO}_{2}$ in a humidified atmosphere for 20 min to allow Matrigel polymerization (Figure 1A). Then, the lower chamber was loaded with $0.75 \mathrm{ml}$ of medium (DMEM or RPMI1640) containing 1\% FBS. In the migration assay, $3 \times 10^{4}$ MIA Paca 2 cells or $1 \times 10^{4}$ PANC- 1 cells in $0.5 \mathrm{ml}$ of serum-free medium were seeded in the upper chamber. In the invasion assay, $5 \times 10^{4}$ of MIA Paca 2 cells or $3 \times 10^{4}$ PANC- 1 cells were seeded. Wells with the same amount of Matrigel in the lower chamber without DRG seeding were used as controls (Matrigel-co). After the confirmation of DRG outgrowth $48 \mathrm{~h}$ after cultivation (Figure 1A), non-migrated or non-invaded cells in the upper chamber were gently removed using cotton swabs. The migrated or invaded cells that passed through the membrane were stained with Diff Quick solution (International Reagents, Japan), and five randomly selected $100 \times$ magnification fields per membrane were counted under an optical microscope. Three wells for each condition were used in one experiment.
Neurotropism assay using a cancer cell-neuron horizontal coculture model. The protocol of the cancer cell-DRG horizontal coculture model was modified as described previously $(12,14)$. Briefly, $5 \times 10^{4}$ of MIA Paca 2 or PANC- 1 cells were suspended in 5 $\mu \mathrm{l}$ of Matrigel drop and placed approximately $1 \mathrm{~mm}$ away from a 5 $\mu \mathrm{l}$ "DRG" Matrigel drop (Figure 2A). To exclude the possibility of unspecific migration of cancer cells, an additional $5 \mu$ "Blank" Matrigel drop was positioned at the opposite site. The dishes were then placed in an incubator set at $37^{\circ} \mathrm{C}$ saturated with $5 \% \mathrm{CO}_{2}$ in a humidified atmosphere for $20 \mathrm{~min}$ to allow for Matrigel polymerization. Each cell-suspended or blank Matrigel was connected with a $1 \mathrm{~mm}$-long Matrigel plug, "Spacer" (Figure 2A). After incubation for additional 20 min for "Spacer" polymerization, the Matrigel drops were carefully submersed in $2 \mathrm{ml}$ of DMEM or RPMI1640 supplemented with 1\% FBS. The co-cultures were incubated at $37^{\circ} \mathrm{C}$ with $5 \% \mathrm{CO}_{2}$ in a humidified atmosphere for 8 days. Representative photographic images of the adjacent and opposite areas of the cancer cell suspension were captured using a microscope (Eclipse TE2000-U, Nikon, Tokyo, Japan) and imaging system (AQUACOSMOS, Hamamatsu Photonics K. K, Shizuoka, Japan). For quantitative analysis of neurotropism in cancer cells, we defined the migration distance of cancer cells towards the DRG as an $\alpha 1$ parameter, and that away from the DRG as $\alpha 2$ parameter, and calculated the cancer neurotropic index as $\alpha 1 / \alpha 2$ ratio (Figure $2 \mathrm{~B}$ ). Images of migrating cancer cells were captured and fused using a microscope (Biozero, KEYENCE, Osaka, Japan), and the distances were measured using ImageJ software (15). This horizontal coculture model was performed in at least five biological replicates.

Preparation of DRG-Conditioned Medium (CM). Twenty DRG cells were isolated from a single mouse and seeded each in a $5 \mu \mathrm{drop}$ of Matrigel on a $60 \mathrm{~mm}$ dish. After DRG suspensions, the dish was incubated at $37^{\circ} \mathrm{C}$ saturated with $5 \% \quad \mathrm{CO}_{2}$ in a humidified atmosphere for $20 \mathrm{~min}$ to allow Matrigel polymerization. Then, 4 $\mathrm{ml}$ of medium (DMEM or RPMI1640) containing 1\% FBS was carefully submersed in the dish and incubated for additional $72 \mathrm{~h}$. After confirming the axonal growth of DRG cells, the supernatant was filtered through a $0.22 \mu \mathrm{m}$ filter (Millex-GV, Merck KGaA, Dermstadt, Germany) and stored at $-30^{\circ} \mathrm{C}$. The medium collected from these steps was used as DRG-conditioned medium (DRG$\mathrm{CM}$ ). CM collected from the dish coated with the same amount of Matrigel without DRGs, was used as a control (Matrigel-CM).

RNA extraction. MIA Paca 2 and PANC- 1 cells $\left(6 \times 10^{4}\right.$ cells $)$ were cultured in $35 \mathrm{~mm}$ dishes with 1\% FBS+DMEM or RPMI 1640. After overnight incubation, the medium was changed to DRG-CM or Matrigel-CM. Total RNA from cells incubated for $48 \mathrm{~h}$ was extracted using an RNAeasy Mini kit (Qiagen, Valencia, CA, USA) according to the manufacturer's protocol. The quality, quantity, and integrity of the total RNA were evaluated using a NanoDrop1000 spectrophotometer (NanoDrop Technologies, Wilmington, DE, USA) and the Agilent 2200 TapeStation System (Agilent Technologies, CA, USA). Samples with an RNA quality (RIN) score of $>8.0$ were processed for RNA-seq. The samples from MIA Paca2 cells treated with DRG-CM and Matrigel-CM were abbreviated as MIA-D and MIA-M, respectively, and those from PANC-1 cells as PAN-D and PAN-M, respectively.

Library preparation and RNA-seq. For preparation of RNA-seq samples, SMARTer Stranded Total RNA Sample Prep Kit - HI 
A

Vertical co-culture model

Matrigel-co DRG-co
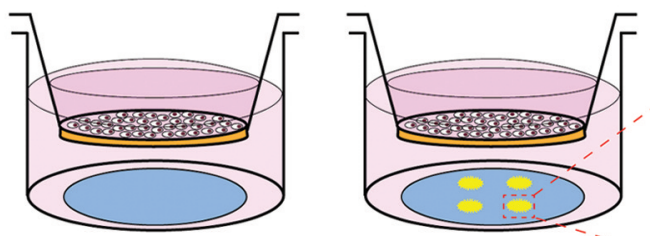

$48 \mathrm{~h}$
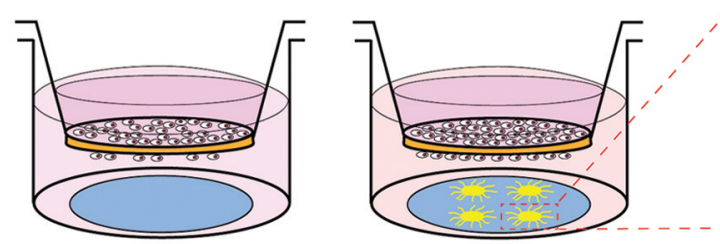

B

Migration
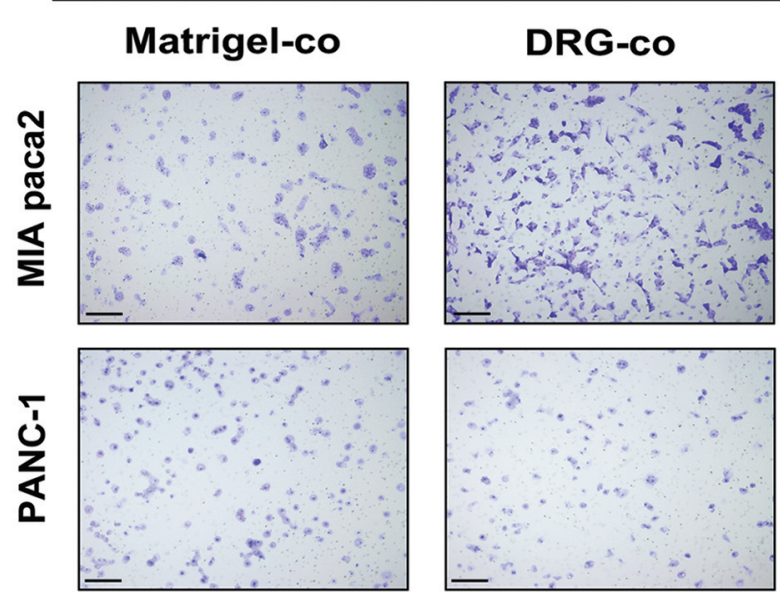

C

Invasion
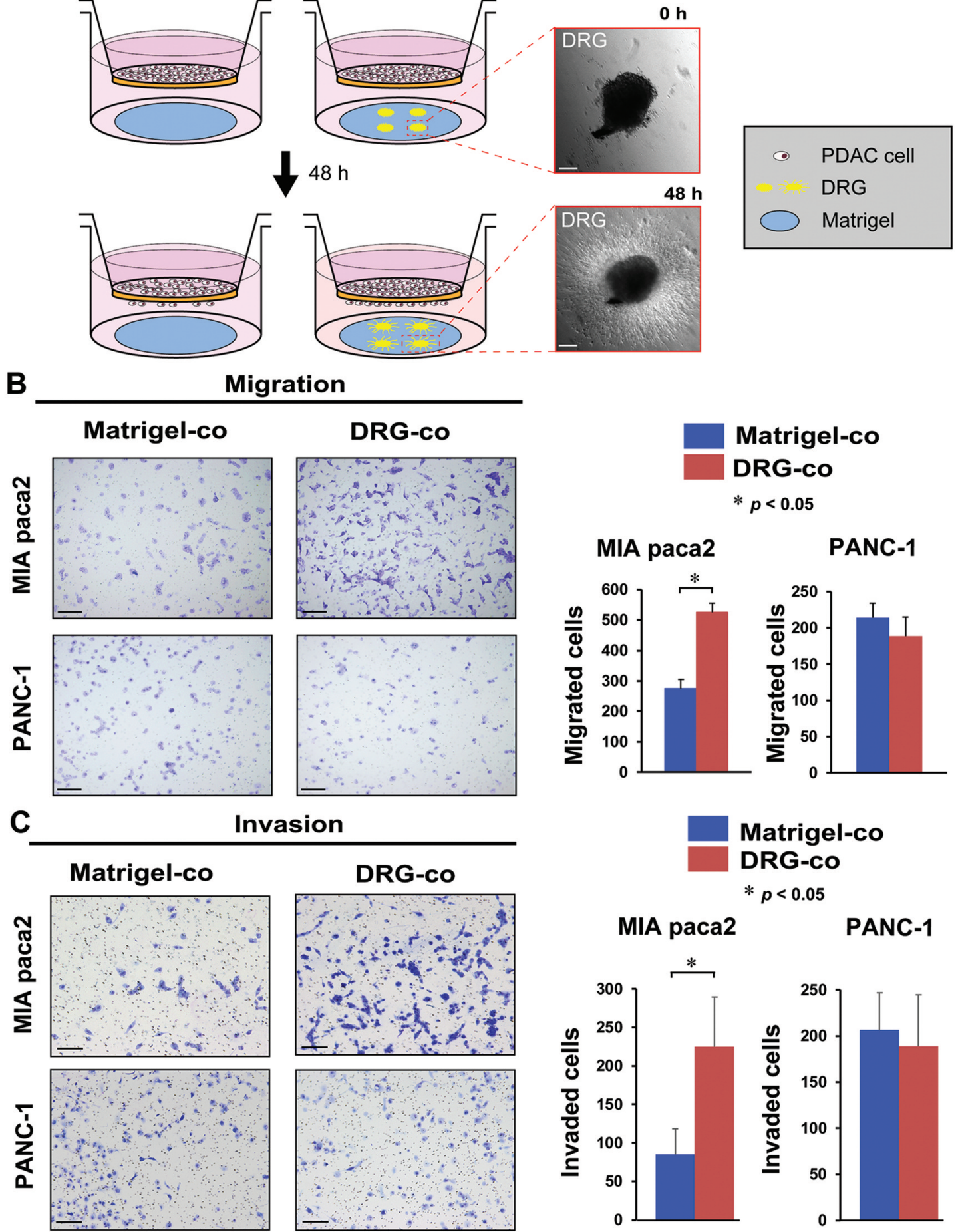

PANC-1

Figure 1. An in vitro vertical co-culture model comprising pancreatic ductal adenocarcinoma cells (PDAC) and mouse dorsal root ganglion (DRG). A) Schematic images of the in vitro vertical co-culture model of a PDAC cell line and DRG. PDAC cells were seeded in the upper transwell chamber and four DRGs were seeded in drops containing $20 \mu \mathrm{l}$ of Matrigel in the lower chamber (DRG-co). After confirming DRG axonal outgrowth after $48 \mathrm{~h}$ of incubation, cells that migrated or invaded through the transwell chamber were counted. Wells with the same number of Matrigel drops without DRG seeding in the lower chamber were used as controls (Matrigel-co). White bar: $200 \mu \mathrm{m} . B, C)$ Migrated (B) and invaded (C) PDAC cells were captured and counted. DRG: Dorsal Root Ganglion; Matrigel-co: Matrigel co-culture model; DRG-co: DRG co-culture model, scale bars: 200 um, *p<0.05. 


\section{Horizontal co-culture model}

A
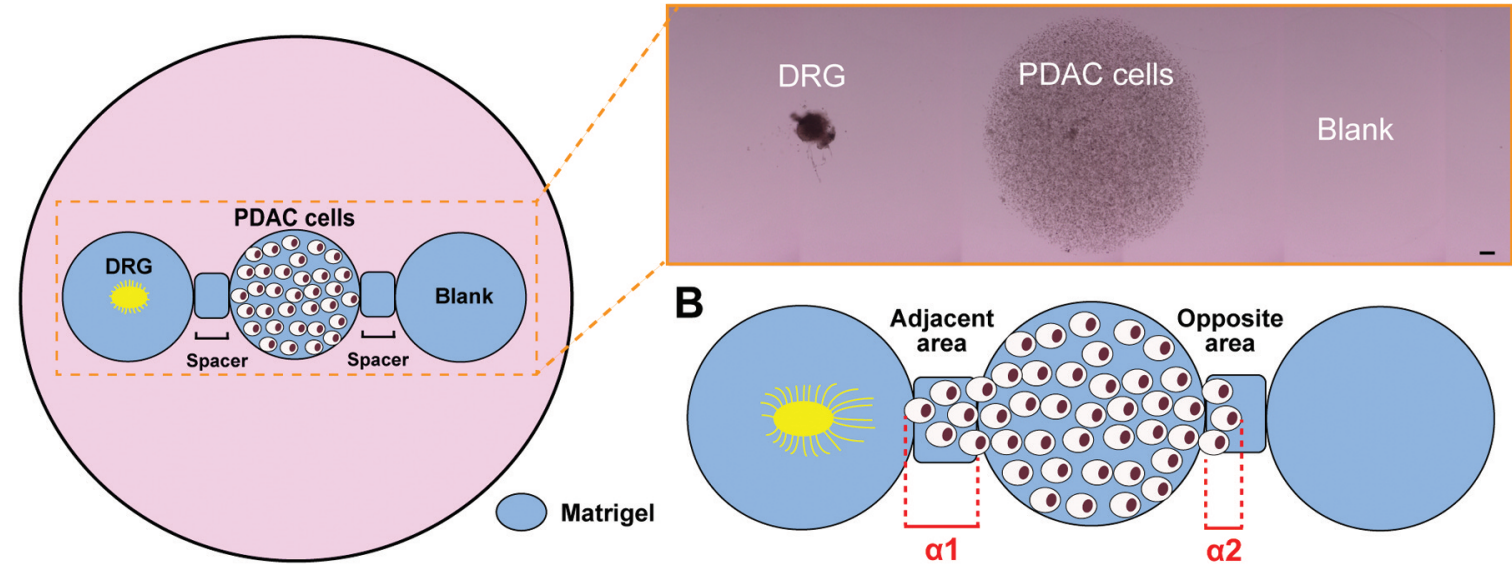

C

MIA Paca2

D

PANC-1
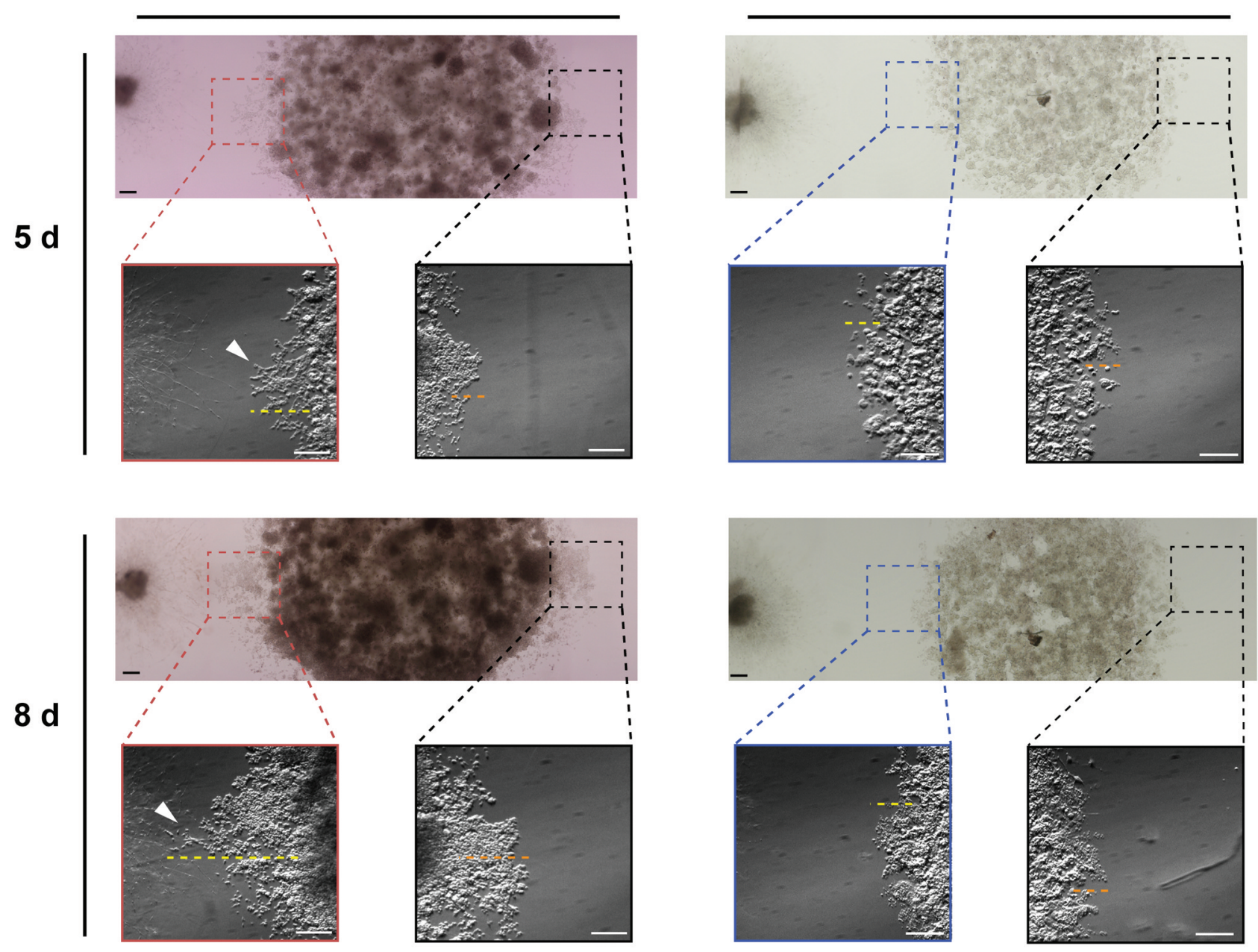

Figure 2. Continued 

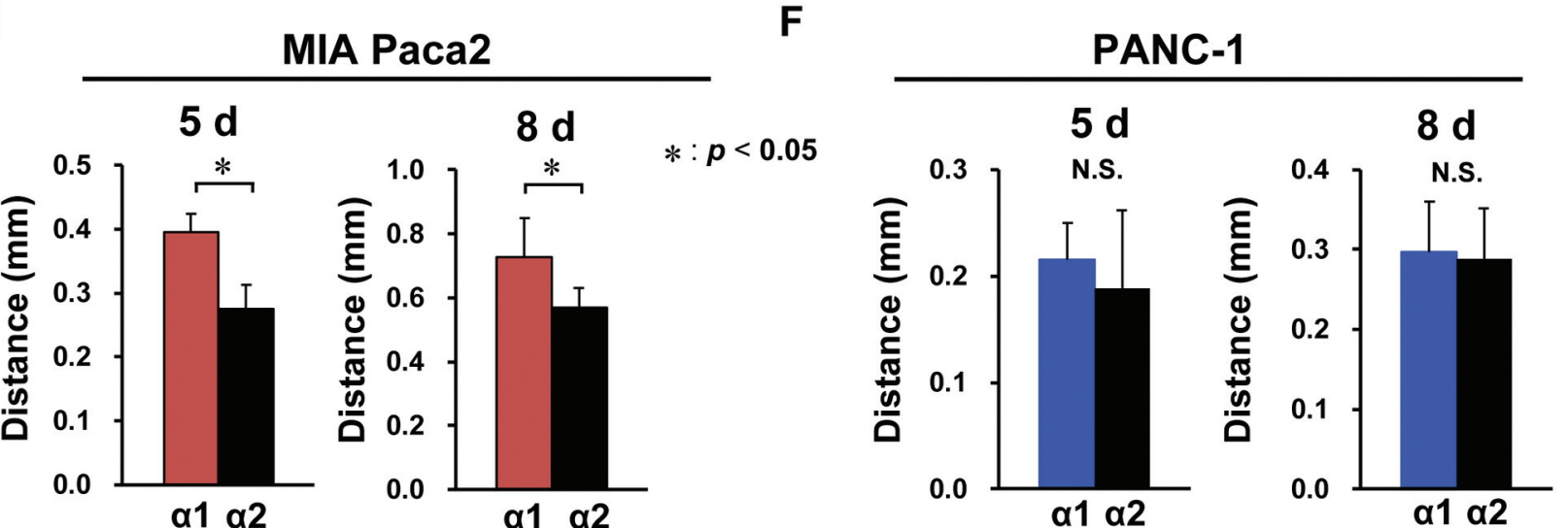

Figure 2. An in vitro horizontal co-culture model with pancreatic ductal adenocarcinoma cells (PDAC) and mouse Dorsal Root Ganglion (DRG). A) Schematic images of the in vitro horizontal co-culture model with a PDAC cell line and DRG. B) An illustration showing the distance covered by migrated tumor cells toward DRG ( $\alpha 1)$ and that away from the DRG toward neurite outgrowth $(\alpha 2)$. C, D) Representative photos of in vitro horizontal co-culture models using PDAC cell lines (C: MIA Paca2 cells, D: PANC-1 cells) and DRG, which were co-incubated for 5 and 8 days, respectively. Magnified micrographs of adjacent and opposite areas are shown. E, F) The distance migrated by the PDAC cells (E: MIA Paca2 cells, F: PANC-1 cells) toward DRG $(\alpha 1)$ and away from DRG $(\alpha 2)$ were calculated (biological replicate, $n=6)$, respectively. [Black bar in fusion: $200 \mu \mathrm{m}$, white bar in magnifying view: $300 \mu \mathrm{m}$, yellow dotted line: the migrated distance or PDAC cells toward DRG ( $\alpha 1)$, orange dotted line: the migrated distance or PDAC cells away from DRG]. d: Days.

Mammalian (Takara Bio, Shiga, Japan) was used according to the manufacturer's protocol. The libraries were sequenced on an Illumina NextSeq 500 instrument (San Diego, CA, USA) with 75 bp $\times 2$ paired end protocol. In total, 12 libraries from each conditioned cell line (3 samples for each MIA-M, MIA-D, PAN-M, PAN-D) were sequenced.

RNA-seq data processing. Gene expression was normalized by the fragments per kilobase of exon per million mapped fragments mapped (FPKM) method and filtered as previously described (16). Principal component analysis (PCA) mapping was performed using the $\log 2$ FPKM value in the $\mathrm{R}$ software (17). Differentially expressed genes (DEGs) with statistical significance were identified through the following filtering steps: the default threshold was $\log _{2}$ (FoldChange) $\geq 1$ and $\mathrm{q}$ value $<0.1$ (false discovery rate was adjusted with the Benjamini-Hochberg method). The Kyoto Encyclopedia of Genes and Genomes (KEGG) pathway analyses were performed with the DAVID bioinformatics tool (version 6.8) (18). $p$-Values were obtained using Fisher's exact test. Raw data analysis was performed by TaKaRa, Inc.

Quantitative RT-PCR. Total RNA was extracted from cultured cells as described above, and reverse transcription was performed using the Primer script RT Reagent kit (Takara Bio, Otsu, Japan) according to the manufacturer's protocol. The cDNA was amplified by quantitative RT-PCR (qRT-PCR) on a Thermal Cycler Dice Real Time System II (Takara Bio) using the Thunderbird 1PCR Mix (Toyobo Life Science, Osaka, Japan). All PCR reactions were run in triplicates, and the relative levels of gene expression normalized to the control was calculated using 2nd Derivative Maximum methods. Sequences of primers used for amplification were as follows: EphA4 (Forward: 5'-TGCACCAAATCAAGTAGCTTCACC-3', Reverse: 5'-CCAGCAGTGTAGCGAGCACAA-3'), $\beta$-actin (Forward: 5'-
TGGCACCCAGCACAATGAA-3', Reverse: 5'-CTAAGTCATA GTCCGCCTAGAAGCA-3'). mRNA levels were normalized to those of $\beta$-actin.

Western blotting. Cells were lysed in chilled lysis buffer supplemented with complete protease and phosphatase inhibitor cocktail (Roche, Basel, Switzerland). Protein concentrations were determined using a bicinchoninic acid protein assay kit (Takara Bio). Protein extracts were subjected to $9 \%$ polyacrylamide gel electrophoresis followed by electroblotting onto an Immobilon-Polyvinylidene fluoride membrane (Millipore, Billerica, MA, USA). After blocking for $30 \mathrm{~min}$ with 5\% skim milk, the membranes were incubated at $4^{\circ} \mathrm{C}$ overnight with primary antibodies including anti-EPHA4 (1:1,000, Invitrogen, $4 \mathrm{C} 8 \mathrm{H} 5)$ and anti- $\beta$-actin $(1: 1,000$, No 5125, Cell Signaling Technology, Danvers, MA, USA). The next day, the membranes were incubated for $1 \mathrm{~h}$ at room temperature with horseradish peroxidaseconjugated secondary antibodies. Immunoreactive bands were visualized using enhanced chemiluminescence plus western blotting detection reagent ((GE Healthcare, Little Chalfont, UK)), and fusion software (Vilber-Lourmat, Collégien, France).

Small interfering RNA inhibition assay. To knockdown endogenous EPHA4 expression in MIA Paca2 cells, we used Stealth RNAi (Invitrogen, Carlsbad, CA, USA). Stealth RNAi Negative Control Medium GC DUPLEX (Invitrogen) was used as a negative control. MIA Paca2 cells and PANC-1 cells were seeded and transfected with Lipofectamine RNAiMAX transfection reagent (Invitrogen), according to the manufacturer's instructions. The MIA Paca2 cells were harvested 2 days after transfection.

Statistical analysis. The in vitro experiments were independently performed at least three times. The data were analyzed using the Student's $t$-test and Mann-Whitney $U$-test for parametric and non- 

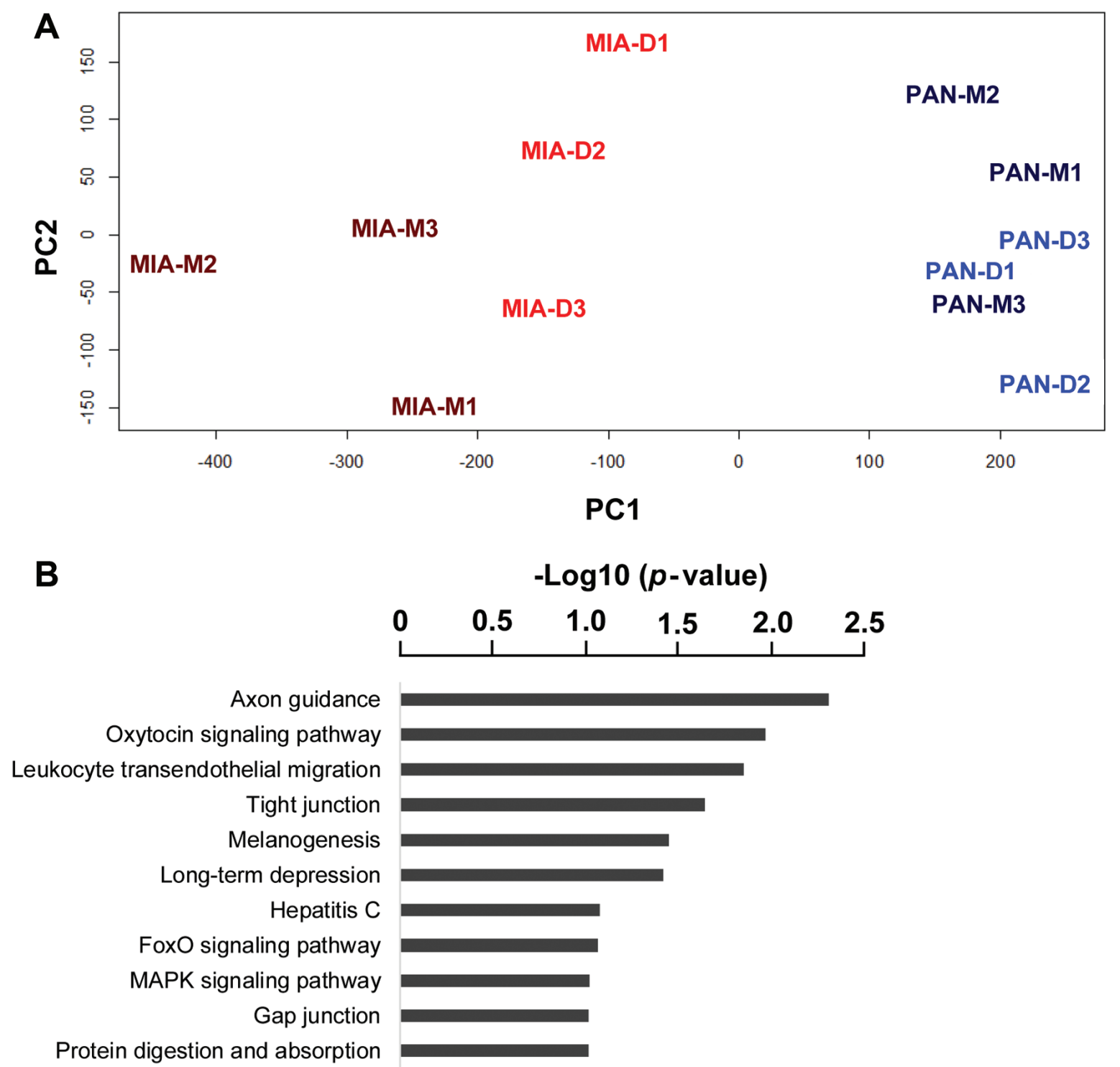

Figure 3. Continued

parametric variables, respectively. $p$-Values $<0.05$ were considered statistically significant. All calculations were performed using SPSS 24.0 software (SPSS Inc., IL, USA).

\section{Results}

Migration and invasion abilities of MIA Paca2 but not PANC1 cells were increased in in vitro vertical co-culture models. Initially, we investigated the migration and invasion abilities of cancer cells toward neurons using in vitro vertical coculture models (Figure 1A). The number of migrated and invaded MIA Paca2 but not PANC-1 cells were significantly increased in the DRG co-culture model (DRG-co) compared to those in the control model (Matrigel-co) (Figure 1B and C).
MIA Paca2 but PANC-1 cells migrated toward DRG neurites in the in vitro horizontal model. Next, we investigated the neurotropism of PDAC cells using an in vitro horizontal cancer-neuron co-culture model (Figure 2A and B). In MIA Paca2 cells, cancer cell colonies extended toward the DRG, and longer extensions of cancer cell colonies were observed in the adjacent area of the DRG compared to the opposite side at 5 and 8 days after cocultivation (Figure 2C). Quantitative analysis showed that the extended distance of MIA Paca2 cells toward the DRG was significantly longer than that toward the blank Matrigel. Conversely, such neurotropic behavior was not observed in PANC-1 cells (Figure 2D). 
C

\section{Up-regulated genes in MIA-M compared with PAN-M}

\begin{tabular}{|c|c|c|}
\hline Name & $p$ value & Log $_{2} \mathrm{FC}$ \\
\hline EPHA4 & 0.0002 & 7.063 \\
\hline SEMA4D & 0.0003 & 1.420 \\
\hline DPYSL5 & 0.0322 & 6.646 \\
\hline SEMA3A & 0.0358 & 6.720 \\
\hline EFNB3 & 0.0967 & 1.613 \\
\hline SEMA6B & 0.1114 & 4.661 \\
\hline RGS3 & 0.1372 & 4.035 \\
\hline ROBO3 & 0.1783 & 3.307 \\
\hline SEMA3D & 0.1878 & 2.473 \\
\hline GNAI3 & 0.2751 & 2.432 \\
\hline SEMA4C & 0.3813 & 2.120 \\
\hline SRGAP3 & 0.4226 & 1.201 \\
\hline SEMA3B & 0.4226 & 2.297 \\
\hline EPHA7 & 0.4226 & 1.599 \\
\hline
\end{tabular}

E

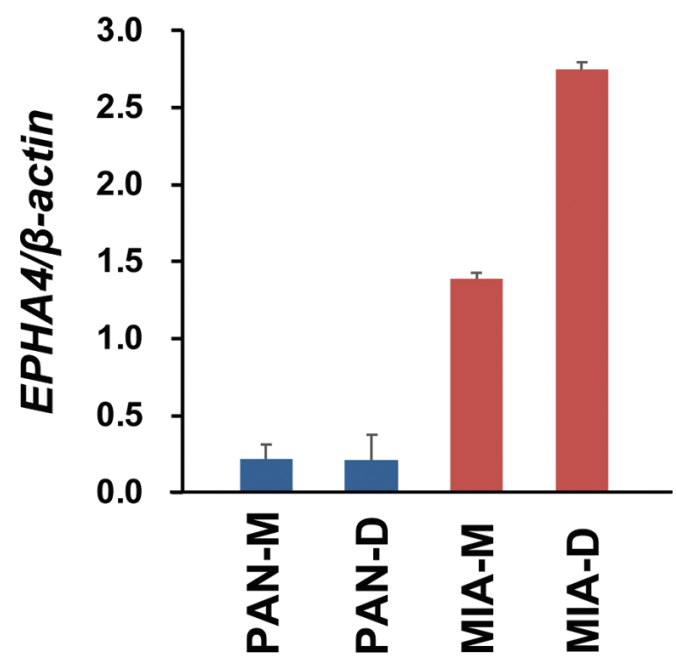

D

\section{Up-regulated genes in MIA-D compared with MIA-M}

\begin{tabular}{|c|c|c|}
\hline Name & $p$ value & Log 2 FC \\
\hline EFNA1 & 0.0008 & 7.741 \\
\hline PAK6 & 0.0112 & 5.542 \\
\hline PLXNB1 & 0.0161 & 3.945 \\
\hline EPHA4 & 0.0164 & 1.002 \\
\hline SEMA3C & 0.0808 & 5.804 \\
\hline ABLIM2 & 0.1837 & 2.572 \\
\hline RHOD & 0.1843 & 1.067 \\
\hline PLXNB3 & 0.2574 & 2.016 \\
\hline NGEF & 0.2748 & 3.483 \\
\hline SEMA4B & 0.3453 & 1.374 \\
\hline EFNA2 & 0.3841 & 1.829 \\
\hline SEMA7A & 0.4226 & 2.003 \\
\hline SEMA3F & 0.4226 & 1.994 \\
\hline UNC5A & 0.4226 & 1.775 \\
\hline EPHB3 & 0.4226 & 1.773 \\
\hline UNC5B & 0.4226 & 1.490 \\
\hline SEMA3G & 0.4226 & 1.667 \\
\hline FES & 0.5806 & 2.159 \\
\hline
\end{tabular}

\section{F Western-blotting}

\section{EPHA4}

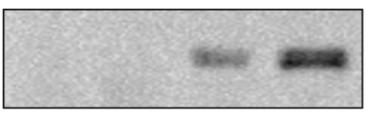

$\beta$-actin

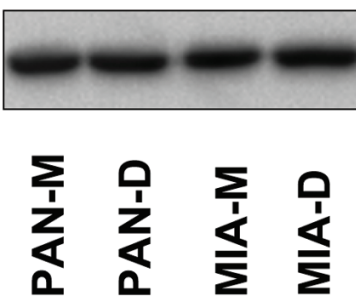

Figure 3. Transcriptome analysis of PDAC cell lines by RNA sequencing. A) Principal component analysis of gene expression of MIA Paca2 cells and PANC-1 cells treated with Dorsal Root Ganglion-conditioned medium (DRG-CM) or Matrigel-conditioned medium (Matigel-CM) (MIA-M1, M2, -M3=MIA Paca2-Matrigel-CM, MIA-D1, -D2, -D3=MIA Paca2-DRG-CM, PAN-M1, -M2-, M3=PANC-1-Matrigel-CM, PAN-D, -D2, D3=PANC1-DRG-CM). B) KEGG pathway analysis with genes that showed significantly different expression levels between MIA-M and PAN-M (the default threshold was Log2 [FoldChange] $\geq 1$ and $q$ value $<0.1)$. C) List of genes belonging to the axon guidance pathway that were up-regulated in MIA Paca2 cells (MIA-M) compared with PANC-1 cells (PAN-M). D) List of genes belonging to the axon guidance pathway that were up-regulated in MIA Paca2 cells (MIA-D) compared to MIA Paca2 cells (MIA-M). E) Quantitative RT-PCR (E) and western blot analyses $(F)$ of EPHA4 expression in PANC-1 and MIA Paca2 cells treated with/without DRG-conditioned medium are shown. 
A

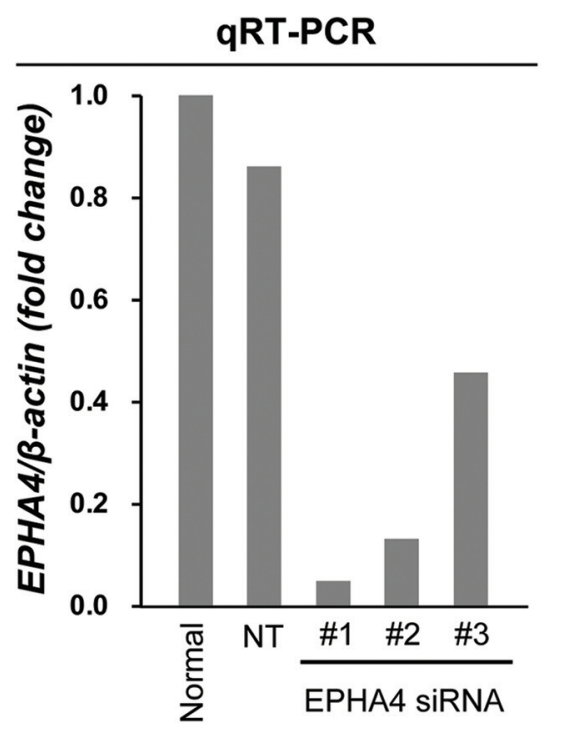

C

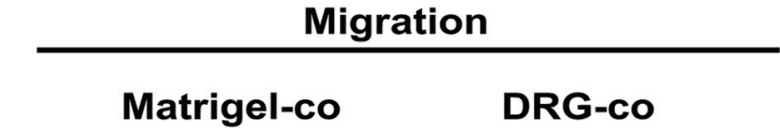

々
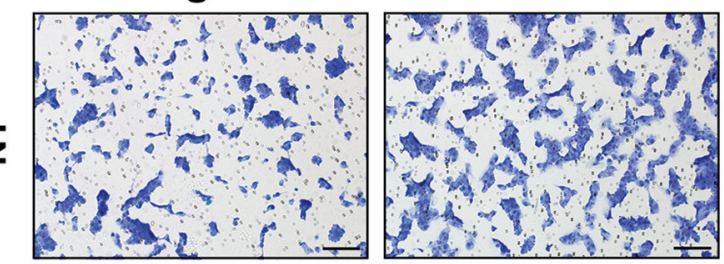

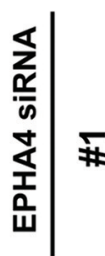
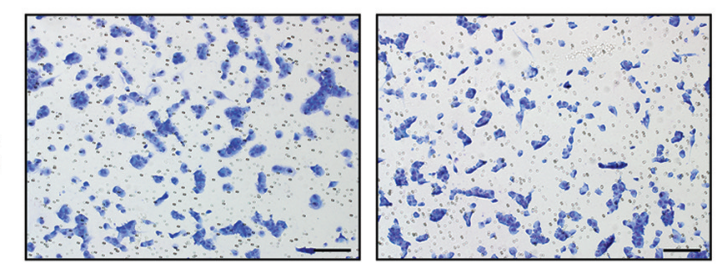

D

\section{Invasion}

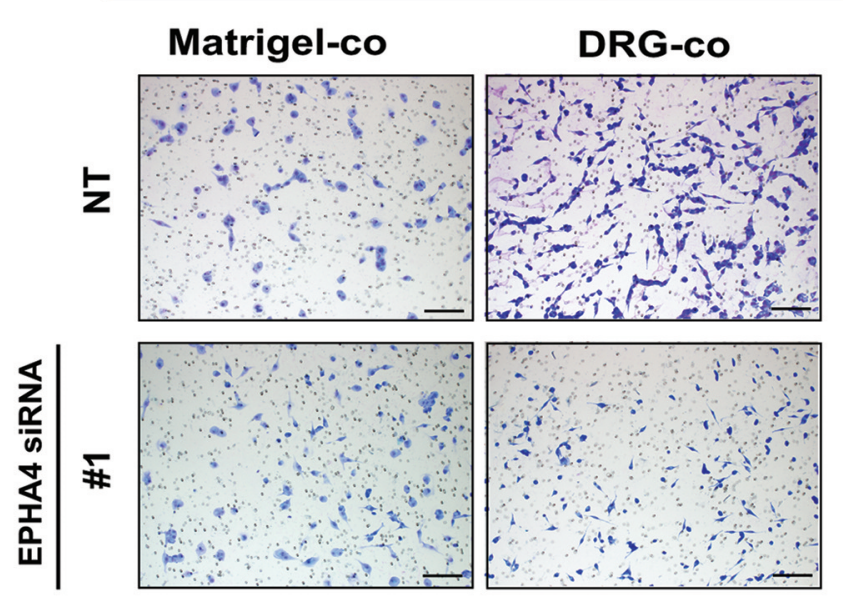

B

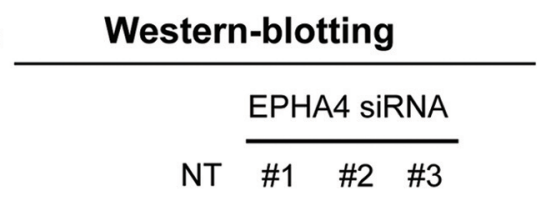

EPHA4

$\beta$-actin
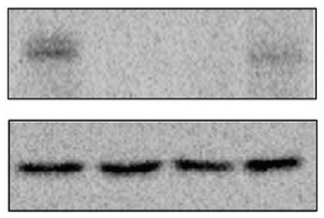



Matrigel-co
DRG-co

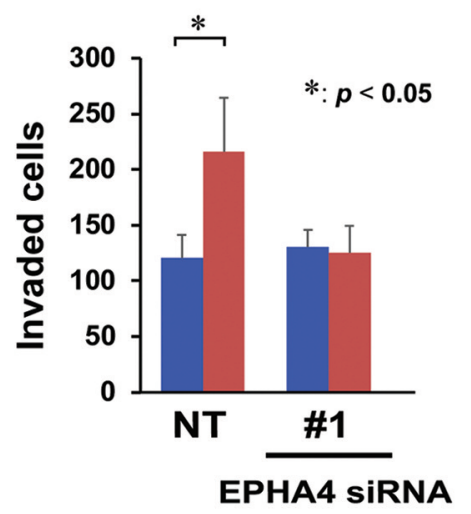

Figure 4. Continued 
E NT
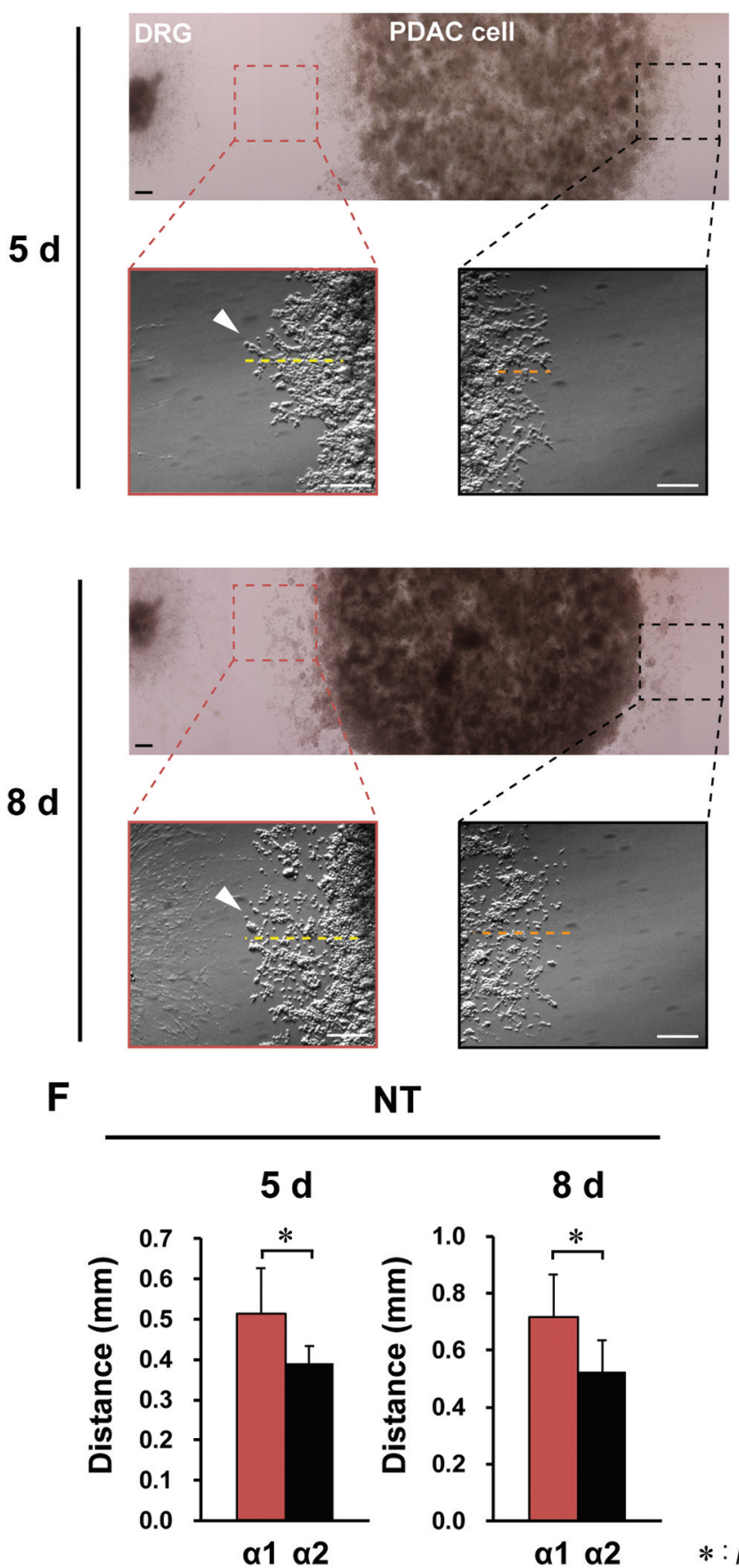

MIA Paca2 cell

EPHA4 SIRNA \#1
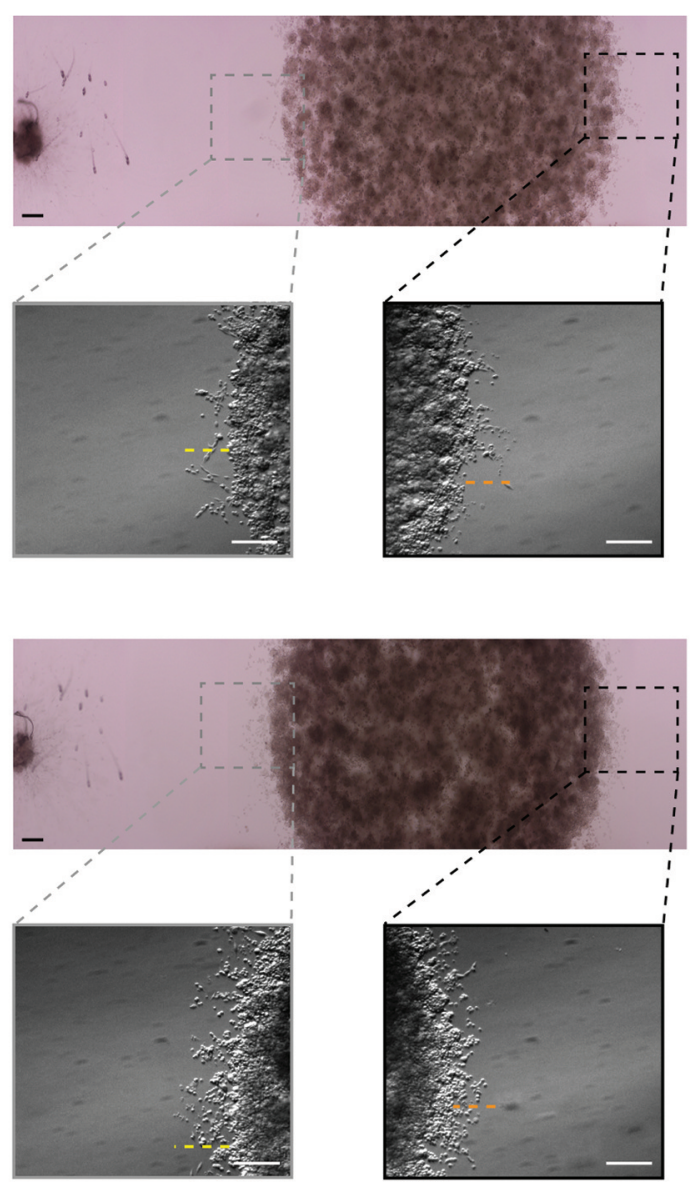

EPHA4 SIRNA \#1

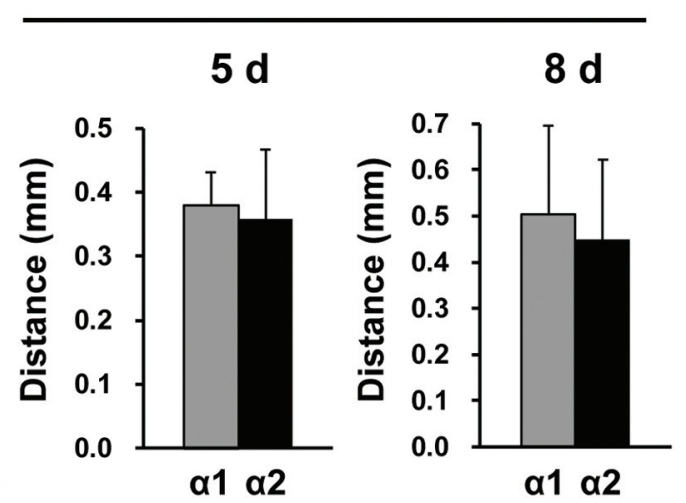

Figure 4. Quantitative RT-PCR (A) and western blot (B) analyses of cells transfected with siRNAs against EPHA4 or with a non-targeting (NT) control siRNA. Migration $(C)$ and invasion (D) assay of MIA Paca2 cells co-cultured with/without DRG after siRNA transfection (2 days). The migrated and invaded cells were captured and counted. E) Representative images of the in vitro horizontal co-culture model using DRG and MIA Paca2 cells after siRNA transfection (2 days), which were incubated for 5 and 8 days, respectively. Magnified micrographs of adjacent and opposite areas are shown. $F)$ The migrated distance of MIA Paca2 cells toward DRG $(\alpha 1)$ and that away from DRG $(\alpha 2)$ were calculated (biological replicate, $n=6)$, respectively. Black bar in fusion: $200 \mu \mathrm{m}$, white bar in magnifying view: $300 \mu \mathrm{m}$, yellow dotted line: the distance migrated by PDAC cells toward DRG ( $\alpha 1)$, orange dotted line: the distance migrated by PDAC cells away from DRG. D: Days; DRG: dorsal root ganglion; PDAC: pancreatic ductal adenocarcinoma. 
Transcriptome signature of PDAC cell lines treated with $D R G-C M$ or Matrigel-CM. Next, comprehensive gene expression in both PDAC cell lines (MIA Paca2 and PANC-1 cells) treated with/without DRG-conditioned medium was profiled using RNA-seq analysis. In total, 27,340 genes were identified, and principal component analysis (PCA) mapping showed that the two cell lines had remarkably distinct gene expression profiles (Figure 3A). This PCA map also revealed a separation of samples according to the culture treated with Matrigel-CM or DRG-CM in MIA Paca2 cells; however, these differences were not observed in PANC-1 cells (Figure 3A).

Among the control cells (MIA-M and PAN-M), of the total 15,415 genes analyzed, 285 showed at least 2-fold differences in expression between the two groups $(p<0.05$, q value $<0.1)$, with 95 genes up-regulated and 190 down-regulated in MIAM compared to PAN-M. Kyoto Encyclopedia of Genes and Genomes (KEGG) pathway analysis of differentially expressed genes between MIA-M and PAN-M showed that genes involved in the regulation of five signaling pathways, including axon guidance, oxytocin signaling, leukocyte transendothelial migration, tight junction, melanogenesis, and long-term depression were significantly enriched (Figure 3B).

Next, we compared gene expression profiles between MIA-M and MIA-D to identify the genes modulated by DRG-inducing signals. Between MIA-D and MIA-M cells, of the total 14,593 genes analyzed, 275 showed at least 2-fold differences in expression between the two groups $(p<0.05)$, with 194 up-regulated and 81 down-regulated genes in MIAD compared to MIA-M. Among the up-regulated genes in MIA-M compared with PAN-M (Figure 3C) and those in MIA-D compared with MIA-M (Figure 3D) that belonged to the axon guidance pathway, we focused on EPHA4 as a predicted target gene responsible for PNI in PDAC cells. qRT-PCR and western blot analyses revealed that the expression of EPHA4 mRNA and EPHA4 protein was elevated in MIA Paca2 cells treated with DRG-CM compared to that in cells treated with Matrigel-M, whereas EPHA4 was faintly expressed in PANC-1 cells (Figure 3E and F).

EPHA4 silencing abrogates neurotropism in a PDAC cell line. To examine the role of EPHA4 in the in vitro co-culture model, we silenced EPHA4 gene expression in the MIA Paca2 cell line using siRNAs (Figure 4A and B). EPHA4 knockdown abolished the migration and invasion abilities of MIA Paca2 cells that were enhanced in the presence of DRG (Figure 4C and D). Furthermore, in the horizontal co-culture model, the migration of MIA Paca2 cells toward the DRG was reduced by knockdown of EPHA4 (Figure 4E and F).

\section{Discussion}

Various mechanisms have been proposed for the development of PNI in cancer cells. Recent studies have proposed the concept of neuron-cancer cell crosstalk; nerve cells have been shown to infiltrate the tumor microenvironment and actively stimulate cancer cell growth by releasing neurotransmitters. In turn, the secretion of neurotrophic growth factors by cancer cells drives the outgrowth of neurons in solid tumors (10). This reciprocal interaction between neurons and cancer cells provides new insights into identifying the novel molecular mechanisms involved, and finally leads to the development of anti-neurogenic therapies. In this regard, the in vitro cancer cell-neuron co-culture models utilized in the present study can provide a proper simulation of the cancer-neuron crosstalk, which evaluates the neurotropism of cancer cells by placing each cell type separately and observing the abilities of cancer cells to migrate and invade in a timedependent manner. This co-culture model has been widely accepted to mimic the conditions of PNI in vivo as described in various studies $(14,19,20)$.

The present study showed that the migratory and invading abilities of MIA Paca2 cells were enhanced in the presence of DRG cells in a vertical co-culture model, as shown in Figure 1. These results indicated that the aggressive nature of MIA Paca2 cells was enhanced by certain soluble factors released by the cancer cell-neuron co-culture. Next, using the horizontal co-culture model shown in Figure 2, we found a significant migration of MIA Paca 2 cells toward neurons. This implies that nerve cells have the potential to determine the polarity of cancer cells by releasing soluble factors in the tumor-neuron microenvironment. Interestingly, this neurotropism of cancer cells was only seen in MIA Paca2, but not in PANC-1 cells.

We then performed a comprehensive transcriptome analysis using MIA Paca2 and PANC-1 cells with/without DRG-CM treatment. This analysis was conducted as follows: 1. to clarify the basic differences in the molecular profiles of MIA Paca2 and PANC-1 cells, especially the potential to receive the signals from the soluble factors released by neurons; and 2. to investigate the molecular changes of MIA Paca2 cells induced by neurons.

PCA mapping using a total of 27,340 genes showed that each cell line had a distinct gene expression profile (Figure $3 \mathrm{~A}$ ), which reflected the differences in their origins. In the KEGG pathway analysis of genes that were significantly differentially expressed between MIA-M and PAN-M cells, axon guidance genes were the most enriched. Axon guidance signaling pathways play important roles in normal neuronal migration and positioning during embryonic development (21). Recent studies have suggested that they have also been implicated in cancer cell growth, survival, invasion, and angiogenesis (22). Furthermore, Jurcak et al. reported that the genes involved in axon guidance can be involved in the regulation of PNI in PDAC (23). Their previous reports are in line with our result that genes in the axon guidance pathway are responsible for the neurotropism of cancer cells 
that lead to PNI. Among genes in the axon guidance pathway, we focused on EPHA4 as a target gene related to PNI, which was a significantly upregulated receptor gene in MIA Paca2 cells treated with DRG-CM compared with the control treatment (Figure 3C and D).

EPHA4 is a member of the erythropoietin-producing hepatocellular (Eph) family of receptor tyrosine kinases. Eph receptors are classified into two subfamilies, type A and B, according to their binding affinities for Ephrin ligands that are categorized into two subclasses, glycosylphosphatidylinositol anchor (A type) and transmembrane domain (B type) (24). These Eph receptors and Ephrin ligands have been implicated in a variety of biological functions, including axon guidance and migration of neural crest cells in the nervous system and establishment of segmental boundaries (25). EphA receptors mainly bind Ephrin A ligands and induce bi-directional cellto-cell contact signaling pathways, such as adhesion, migration, and invasion by modifying the organization of the actin cytoskeleton and influencing the activities of integrins and intercellular adhesion molecules (26). In cancer cells, Eph-Ephrin signals can function as both tumor promoters and suppressors (26). In certain cellular contexts, Eph receptors activated by ephrins have lost the ability to suppress tumorigenicity, and have acquired oncogenic ability (26). In addition, in terms of ligands of the Eph receptor, there exists a cleaved, soluble form of Ephrin molecule that can provide longer-range functions (27). Wycosky et al. have demonstrated that Ephrins function as soluble, monomeric factors during cancer maintenance and/or progression as well as in normal developmental/physiological processes (28). In this study, EPHA4 knockdown by siRNA in MIA Paca2 cells prevented their migration, invasion, and neurotropic abilities, which were enhanced in the presence of neurons (Figure 4). These results and those of previous studies supported our speculation that the presence of certain soluble forms of ligands, such as Ephrin A, in the tumor-nerve microenvironment, enhanced EPHA4 expression in MIA Paca2 cells, resulting in the migration of cancer cells toward neurons, which finally leads to the establishment of PNI.

This study has several limitations. First, we did not investigate the upstream factors that enhanced EPHA4 expression. As upstream molecules, soluble forms of Ephrin or some other proteins bound to EPHA are relevant candidates. Comprehensive analysis of the conditioned medium extracted from a cancer cell-neuron co-culture model is warranted. Second, the clinical impact of EPHA4 expression in PDAC is lacking in this study. Previous studies have suggested that EPHA4 overexpression was related to poor prognosis and metastasis in PDAC cells $(29,30)$. However, no reports have shown the clinicopathological relationships between PNI and EPHA4 expression. Immunohistochemical analysis or in situ hybridization studies using clinical samples are needed to confirm the clinical importance of our study.
In conclusion, we investigated the mechanisms of PNI using a cancer cells-nerve cells co-culture model. EPHA4 can be a target molecule that regulates PNI in PDAC.

\section{Conflicts of Interest}

All Authors have no conflicts of interest to disclose in relation to this study.

\section{Authors' Contributions}

S.F.: design of the work, data acquisition, data analysis, drafting the work. Y.M.: design of the work, drafting the work. S.I.: drafting the work. R.M.: data acquisition, drafting the work. R.K.: drafting the work. M.T.: drafting the work. H.K.: drafting the work. Y.H.: drafting the work. KK: drafting the work. M.S.: drafting the work. All Authors read and approved the final manuscript.

\section{Acknowledgements}

This study was supported in part by the Ministry of Education, Culture, Sports, Science, and Technology of Japan grants-in-aid 17K10694 (Y. Morita) and 19K09167 (H. Kikuchi). This work was also supported by the MEXT/Japan Society for the Promotion of Science KAKENHI (grant number JP15H05898B1) and the Imaging Platform supported by the Ministry of Education, Culture, Sports, Science and Technology (MEXT), Japan, and AMED (grant number JP18gm0910004). The Authors thank Aya Kitamoto, Noritaka Masaki, and other laboratory members for their technical supports.

\section{References}

1 Siegel R, Miller K and Jemal A: Cancer statistics, 2018. CA: A Cancer Journal for Clinicians 68(1): 7-30, 2019. DOI: $10.3322 /$ caac. 21442

2 Wolfgang CL, Herman JM, Laheru DA, Klein AP, Erdek MA, Fishman EK and Hruban RH: Recent progress in pancreatic cancer. CA Cancer J Clin 63(5): 318-348, 2013. PMID: 23856911. DOI: $10.3322 /$ caac. 21190

3 DiMagno EP, Reber HA and Tempero MA: AGA technical review on the epidemiology, diagnosis, and treatment of pancreatic ductal adenocarcinoma. American Gastroenterological Association. Gastroenterology 117(6): 1464-1484, 1999. PMID: 10579989. DOI: 10.1016/s0016-5085(99)70298-2

4 Amit M, Na'ara S and Gil Z: Mechanisms of cancer dissemination along nerves. Nature Reviews Cancer 16(6): 399408, 2019. DOI: $10.1038 /$ nrc. 2016.38

5 Shimada K, Nara S, Esaki M, Sakamoto Y, Kosuge T and Hiraoka N: Intrapancreatic nerve invasion as a predictor for recurrence after pancreaticoduodenectomy in patients with invasive ductal carcinoma of the pancreas. Pancreas 40(3): 464468, 2021. DOI: 10.1097/MPA.0b013e31820b5d37

6 Ceyhan GO, Bergmann F, Kadihasanoglu M, Altintas B, Demir IE, Hinz U, Müller MW, Giese T, Büchler MW, Giese NA and Friess H: Pancreatic neuropathy and neuropathic pain - a comprehensive pathomorphological study of 546 cases. Gastroenterology 136(1): 177-186.e1, 2009. PMID: 18992743. DOI: $10.1053 /$ j.gastro.2008.09.029 
7 Chatterjee D, Katz MH, Rashid A, Wang H, Iuga AC, Varadhachary GR, Wolff RA, Lee JE, Pisters PW, Crane CH, Gomez HF, Abbruzzese JL, Fleming JB and Wang H: Perineural and intraneural invasion in posttherapy pancreaticoduodenectomy specimens predicts poor prognosis in patients with pancreatic ductal adenocarcinoma. Am J Surg Pathol 36(3): 409-417, 2012. PMID: 22301497. DOI: 10.1097/PAS.0b013e31824104c5

8 Kayahara M, Nakagawara H, Kitagawa $\mathrm{H}$ and Ohta T: The nature of neural invasion by pancreatic cancer. Pancreas 35(3): 218-223, 2007. PMID: 17895841. DOI: 10.1097/mpa.0b013 e3180619677

9 Bockman DE, Büchler $M$ and Beger HG: Interaction of pancreatic ductal carcinoma with nerves leads to nerve damage. Gastroenterology 107(1): 219-230, 1994. PMID: 8020665. DOI: 10.1016/0016-5085(94)90080-9

10 Jobling P, Pundavela J, Oliveira SM, Roselli S, Walker MM and Hondermarck H: Nerve-Cancer Cell Cross-talk: A novel promoter of tumor progression. Cancer Res 75(9): 1777-1781, 2015. PMID: 25795709. DOI: 10.1158/0008-5472.CAN-14-3180

11 Dai H, Li R, Wheeler T, Ozen M, Ittmann M, Anderson M, Wang Y, Rowley D, Younes M and Ayala GE: Enhanced survival in perineural invasion of pancreatic cancer: An in vitro approach. Hum Pathol 38(2): 299-307, 2007. PMID: 17097719. DOI: 10.1016/j.humpath.2006.08.002

12 Furuhashi S, Sakaguchi T, Murakami T, Fukushima M, Morita Y, Ikegami K, Kikuchi H, Setou M and Takeuchi H: Tenascin C in the tumor-nerve microenvironment enhances perineural invasion and correlates with locoregional recurrence in pancreatic ductal adenocarcinoma. Pancreas 49(3): 442-454, 2020. PMID: 32132519 . DOI: 10.1097/MPA.0000000000001506

13 Ayala GE, Wheeler TM, Shine HD, Schmelz M, Frolov A, Chakraborty S and Rowley D: In vitro dorsal root ganglia and human prostate cell line interaction: Redefining perineural invasion in prostate cancer. Prostate 49(3): 213-223, 2001. PMID: 11746267. DOI: 10.1002/pros.1137

14 Li X, Wang Z, Ma Q, Xu Q, Liu H, Duan W, Lei J, Ma J, Wang X, Lv S, Han L, Li W, Guo J, Guo K, Zhang D, Wu E and Xie $\mathrm{K}$ : Sonic hedgehog paracrine signaling activates stromal cells to promote perineural invasion in pancreatic cancer. Clin Cancer Res 20(16): 4326-4338, 2014. PMID: 24947933. DOI: 10.1158/1078-0432.CCR-13-3426

15 Schneider CA, Rasband WS and Eliceiri KW: NIH image to imageJ: 25 years of image analysis. Nat Methods 9(7): 671-675, 2012. PMID: 22930834. DOI: 10.1038/nmeth.2089

16 Kawamura T, Yamamoto M, Suzuki K, Suzuki Y, Kamishima M, Sakata M, Kurachi K, Setoh M, Konno H and Takeuchi H: Tenascin-C produced by intestinal myofibroblasts promotes colitis-associated cancer development through angiogenesis. Inflamm Bowel Dis 25(4): 732-741, 2019. PMID: 30517646. DOI: $10.1093 / \mathrm{ibd} / \mathrm{izy} 368$

17 Team RC: R core team. R: A language and environment for statistical computing. Foundation for Statistical Computing, 2013.

18 Huang da W, Sherman BT and Lempicki RA: Systematic and integrative analysis of large gene lists using DAVID bioinformatics resources. Nat Protoc 4(1): 44-57, 2009. PMID: 19131956. DOI: $10.1038 /$ nprot.2008.211

19 Gil Z, Cavel O, Kelly K, Brader P, Rein A, Gao SP, Carlson DL, Shah JP, Fong $\mathrm{Y}$ and Wong RJ: Paracrine regulation of pancreatic cancer cell invasion by peripheral nerves. J Natl
Cancer Inst 102(2): 107-118, 2010. PMID: 20068194. DOI: 10.1093/jnci/djp456

20 Liebig C, Ayala G, Wilks JA, Berger DH and Albo D: Perineural invasion in cancer: A review of the literature. Cancer 115(15): 3379-3391, 2009. PMID: 19484787. DOI: 10.1002/cncr.24396

21 Russell S and Bashaw G: Axon guidance pathways and the control of gene expression. Developmental Dynamics 247(4): 571-580, 2019. DOI: 10.1002/dvdy.24609

22 Mehlen P, Delloye-Bourgeois C and Chédotal A: Novel roles for Slits and netrins: Axon guidance cues as anticancer targets? Nat Rev Cancer 11(3): 188-197, 2011. PMID: 21326323. DOI: $10.1038 / \mathrm{nrc} 3005$

23 Jurcak NR, Rucki AA, Muth S, Thompson E, Sharma R, Ding D, Zhu Q, Eshleman JR, Anders RA, Jaffee EM, Fujiwara K and Zheng L: Axon guidance molecules promote perineural invasion and metastasis of orthotopic pancreatic tumors in mice. Gastroenterology 157(3): 838-850.e6, 2019. PMID: 31163177. DOI: $10.1053 /$ j.gastro.2019.05.065

24 Gale NW, Holland SJ, Valenzuela DM, Flenniken A, Pan L, Ryan TE, Henkemeyer M, Strebhardt K, Hirai H, Wilkinson DG, Pawson $\mathrm{T}$, Davis $\mathrm{S}$ and Yancopoulos GD: Eph receptors and ligands comprise two major specificity subclasses and are reciprocally compartmentalized during embryogenesis. Neuron 17(1): 9-19, 1996. PMID: 8755474. DOI: 10.1016/s0896-6273(00)80276-7

25 Flanagan JG and Vanderhaeghen P: The ephrins and Eph receptors in neural development. Annu Rev Neurosci 21: 309-345, 1998. PMID: 9530499. DOI: 10.1146/annurev.neuro.21.1.309

26 Pasquale EB: Eph receptors and ephrins in cancer: Bidirectional signalling and beyond. Nat Rev Cancer 10(3): 165-180, 2010. PMID: 20179713. DOI: $10.1038 / \mathrm{nrc} 2806$

27 Cramer KS and Miko IJ: Eph-ephrin signaling in nervous system development. F1000Res 5: 2016. PMID: 27092247. DOI: 10.12688/f1000research.7417.1

28 Wykosky J, Palma E, Gibo DM, Ringler S, Turner CP and Debinski W: Soluble monomeric EphrinA1 is released from tumor cells and is a functional ligand for the EphA2 receptor. Oncogene 27(58): 7260-7273, 2008. PMID: 18794797. DOI: 10.1038/onc. 2008.328

29 Iiizumi M, Hosokawa M, Takehara A, Chung S, Nakamura T, Katagiri T, Eguchi H, Ohigashi H, Ishikawa O, Nakamura Y and Nakagawa H: EphA4 receptor, overexpressed in pancreatic ductal adenocarcinoma, promotes cancer cell growth. Cancer Sci 97(11): 1211-1216, 2006. PMID: 16965393. DOI: 10.1111/ j.1349-7006.2006.00313.x

30 Takano H, Nakamura T, Tsuchikawa T, Kushibiki T, Hontani K, Inoko K, Takahashi M, Sato S, Abe H, Takeuchi S, Sato N, Hiraoka K, Nishihara H, Shichinohe T and Hirano S: Inhibition of Eph receptor A4 by 2,5-dimethylpyrrolyl benzoic acid suppresses human pancreatic cancer growing orthotopically in nude mice. Oncotarget 6(38): 41063-41076, 2015. PMID: 26516928. DOI: $10.18632 /$ oncotarget.5729

Received February 18, 2021

Revised February 27, 2021

Accepted March 1, 2021 